\title{
Ripple transport and neoclassical diffusion in IR-T1 tokamak
}

\author{
M. Hosseininejad ${ }^{1} \cdot$ M. Ghoranneviss ${ }^{1} \cdot$ M. K. Salem ${ }^{1}$
}

Received: 8 June 2019 / Accepted: 21 September 2019 / Published online: 26 November 2019

(c) The Author(s) 2019

\begin{abstract}
In tokamaks, small variations in the magnetic field create ripple. The discontinuous nature of the magnetic field coils in an axisymmetric torus conduces to additional particle trapping, and it is responsible for an additional neoclassical diffusion. Ripples also reduce the particle removal efficiency and disturb plasma confinement and cause constraints in the design of magnet of fusion reactor. Therefore, it is quite important to include the ripple for the design of plasma edge components. Herein, several considerations are taken into account to calculate and evaluate the diffusion coefficient and ion heat conductivity in ripple transport and also to compare it with neoclassical mode.
\end{abstract}

Keywords Neoclassical diffusion · Ripple magnetic field · Ripple transport · Diffusion coefficient · Diffusivity · Ion heat conductivity

\section{Introduction}

The axisymmetry of the magnetic field has been assumed as one of the substantial advantages of tokamaks. Apart from the simplicity of analysis, the radial motion of particles trapped in low collision frequencies is less than that of asymmetric fields [1].

It is expected that in an axisymmetric magnetic field configuration of a tokamak D-T reactor, fusion-produced alpha particles are well confined and thus provide for efficient internal plasma heating. The presence of magnetic perturbations, however, may greatly affect the retention of these particles and lead to their loss from the confinement region [2].

Small variations in the magnetic field are called magnetic ripple. In practice, the discrete nature of the magnetic field of the toroidal coils around the torous creates a ripple in the field intensity with a period equal to the distance between the centers of the coils. This perturbation is toroidal field ripple (TFR).

Ripple has negative effects on fusion plasma performance including the fusion energy reduction due to particle loss, the plasma beta reduction and the rotation break. Generally,

M. Ghoranneviss

ghoranneviss@gmail.com

1 Plasma Physics Research Center, Science and Research Branch, Islamic Azad University, P.O. Box: 14665-678, Tehran, Iran various magnetic perturbations like toroidal field ripples can degrade fast ions confinement. In the outer region of tokamak plasmas due to loose winding of toroidal coils, TF ripples have strongest effect and can cause fast ions to loose very quickly which in turn can also damage the first wall of the tokamak [2-5].

The ripple strength not only enhances the diffusion coefficient but also changes the energy dependence [6].

An ideal, axisymmetric tokamak has no ripple. In real-life machines, the finite widths of the toroidal field coils create a periodic ripple [7].

Deviation from the symmetry is generally considered to be fiddling. This effect will be more important probably in the next generation of machines, for two reasons. It is expected to reach higher temperatures which is accompanied by upsurging of asymmetry effect as the frequency of collision reduces. In some collisional regime for the present and next generation of tokamaks, the ripple diffusion changes inversely to frequency of collision [1].

In "Ripple transport" section, we discuss about the ripple transport and the ripple magnetic field in the toroidal coordinate and superbanana diffusion. In "A solution of the Fokker-Planck equation for trapped particles in the magnetic field ripples" and "Diffusion and heat transport" sections, the Fokker-Planck equation is presented for particles trapped in the ripple magnetic field and the equations for the particle flux and heat flux are obtained to calculate the diffusion coefficient and the ion heat conductivity and finally 
transport coefficients are compered in neoclassical and ripple mode for IR-T1 tokamak.

\section{Ripple transport}

The ripple transport is due to the drift motion of particles distributed in the ripple of a magnetic field. The moderate number of toroidal field coils of a tokamak expunges the perfect axial symmetry of the system. The coils create a short wavelength in the magnetic field strength, namely "ripple," as a field line is pursued around the torus.

For $N$ coils, the magnetic field can be presented by

$B=B_{0}(1-\varepsilon \cos \theta)(1-\delta(r, \theta) \cos N \phi)$

where $\theta$ is the poloidal angle circa the magnetic axis, $\phi$ indicates the angular distance around the axis of symmetry, $r$ is the radial distance from the magnetic axis, $R$ is the radius of the magnetic axis and $\varepsilon=r / R$ is the inverse aspect ratio.

An estimation for the value of $\delta$, for the case of strongly elongated toroidal field coils, is defined by

$\delta=\left(\frac{R}{R_{\text {outer }}}\right)^{N}+\left(\frac{R_{\text {inner }}}{R}\right)^{N}$,

where $R_{\text {outer }}$ and $R_{\text {inner }}$ are, respectively, the major radii of the outer and inner legs of the toroidal coils space [3].

For a particle to be trapped in the ripple wells it has to satisfy the condition that the parallel velocity sufficiently small that magnetic mirror reflection occurs in the ripple well. This is equivalent to the statement that the banana turning point positions of the particles lie inside the ripple well region. Particles are trapped into and re-trapped out of ripple well by collisional processes, in particular by pitchangle scattering, which change the ratio between the parallel and perpendicular velocities. The typical residence time of a particle in ripple well is of the order $\Delta t \sim \delta / v$, where $v$ is the collisional deflection frequency. The fraction of particles which is ripple well trapped is of order $\delta^{1 / 2}$.

Due to the toroidal field gradient drift, the trapped particles in the ripple well drift vertically with a velocity of $v_{d} \sim \rho v / R$ where $\rho$ is the Larmor radius and $v$ is the total particle velocity. Incorporating the ripple well trapped fraction, the drift velocity and the residence time, a diffusion coefficient for collisional ripple well transport can be estimated as:

$D \sim \delta^{1 / 2}\left(\frac{\rho v \delta}{R \nu}\right) \frac{v}{\delta} \sim \frac{\delta^{3 / 2} \rho^{2} v^{2}}{R^{2} v}$

where $D$ is radial diffusion coefficient for a circular plasma, averaged over the pitch angle. This implies a greater loss rate for ions than for electrons, and an ambipolar electric field will evolve to reduce the ion particle diffusion. Nevertheless, Eq. (2) remains an appropriate estimate. It should, however, be noted that its validity is restricted to collision frequencies $v>v_{\mathrm{d}} \delta / a$, where $a$ is plasma minor radius; otherwise, particles would drift out of the plasma before suffering a collision, producing a loss cone in velocity space rather than a diffusion. This restriction is particularly stringent as $D$ which is dominated by contributions from the more energetic particles, so that the inequality must hold for these particles. (Energies are approximately five times the plasma temperature.) The proper poloidal averaging procedure leads to a significant reduction of $D$ from that given in Eq. (2) [3].

The ripple-induced diffusion is different from the superbanana diffusion in stellarators. Stringer has given an ample argument of this difference which becomes evident at small values of $N$ [3]. He showed that for values of $\alpha=\varepsilon t / N \delta$ ( $t$ is the rotational transformation) in excess of unity the ripple does not outcome in the constitution of local magnetic mirrors and resultant trapping over the whole minor azimuth, and that, generally for $\alpha$ of order unity, considerable diminution of the mirror depth arises at all angles. This can be seen from Eq. (1) by noting minimum in $B_{l}$ (where $l$ is arc length along a field line) arise only if $\alpha \sin \theta<1$. Even when this retains, each ripple is an asymmetric mirror with mirror rotation $R_{ \pm}(\theta)$ where

$\left(R_{-}-1\right)<2 \delta<\left(R_{+}-1\right)$

Therefore, a well depth $\Delta(\theta) \equiv\left(R_{-}-1\right)$ is given by

$$
\begin{aligned}
\Delta(\theta)= & 2 \delta\left[\sqrt{1-\alpha^{2} \sin ^{2} \theta}\right. \\
& \left.-\alpha\left\{\frac{\pi}{2}-\sin ^{-1}(\alpha|\sin \theta|)\right\}|\sin \theta|\right]
\end{aligned}
$$

Stringer's estimation of the decrease in diffusion and ion heat flux is attained by including this decrease in the well depth from $2 \delta$ to $\Delta(\theta)$. Though this effect is a highly important factor, it undermines the asymmetric distortion of the ripple wells. In the next part, here we offer the solution of the Fokker-Planck equation for the ripple-trapped distribution, and then we deduced the resultant transport coefficients.

The analysis offered is right in the main range of collision frequencies $v_{j}$ given by

$\frac{\delta^{1 / 2} v_{\mathrm{Th}_{j}}^{N}}{R}>\frac{v_{j}}{\delta}>\frac{v_{\mathrm{Th}_{j}}^{2} \delta}{\omega_{c j} r^{2}}$

where $v_{\mathrm{Th}_{j}}$ is the thermal speed of species $j$ and $\omega_{c j}$ is the gyrofrequency. Physically, it means that the effective collision frequency $v_{\text {eff }}=v / \delta$ is less than the bounce time of a ripple-trapped particle $\omega_{b}=\delta^{1 / 2} v_{\mathrm{Th}} N / R$, but higher than the drift frequency circa of a complete superbanana orbit in the mirror azimuth of the torus [8-11]. 


\section{A solution of the Fokker-Planck equation for trapped particles in the magnetic field ripples}

Collision frequencies in range (4) can be considered by writing a kinetic equation which presumes the jump frequency and effective collision frequency $v_{\text {eff }}=\nu / \delta$ are comparable and then writing a secondary expansion in $v_{\text {eff }} / \omega_{b}$. We define the distribution function $f_{j}$ which is the average amount over a gyro-period of the Larmor radius to a Maxwellian $F_{j}$ that satisfies Eq. (5):

$\sigma q \vec{n} \cdot \nabla f_{j}+\vec{v}_{d j} \cdot \nabla F_{j}=C\left(f_{j}\right)$

where

$F_{j}=\left(\frac{m_{j}}{2 \pi T_{j}(r)}\right)^{3 / 2} n_{j}(r) \exp \left(\frac{m_{j} \kappa}{T_{j}(r)}\right)$

And the kinetic energy per unit mass is $\kappa=v^{2} / 2$, and $\sigma$ is the sign of the velocity $q$ along the field. The gradient operators in Eq. (5) are taken into account at constant energy

$W=\frac{v^{2}}{2}+\frac{e_{j} \Phi(r)}{m_{j}}$

where $\Phi(r)$, as a function of radius, is an electrostatic potential. The drift velocity $\vec{v}_{d j}$ is given by

$\vec{v}_{d j}=\frac{1}{\omega_{c_{j}}} \vec{n} \Lambda\left(\mu \nabla B+q^{2} \vec{n} \cdot \nabla \vec{n}\right)-\frac{\nabla \Phi \Lambda B}{B^{2}}$

where $\vec{n}$ is a unit vector along the field and the magnetic moment is expressed by $\mu$. We know $\kappa=q^{2} / 2+\mu B$.

To acquire a dissolvable equation for $f_{j}$, we exchange the $C\left(f_{j}\right)$ by an ordinary pattern. For electron-ion collision, we can apply the Lorentz approximation

$C_{\mathrm{ei}}\left(f_{\mathrm{e}}\right)=v_{\mathrm{ei}}(\kappa) \frac{q}{B} \frac{\partial}{\partial \mu} q \mu \frac{\partial}{\partial \mu} f_{\mathrm{e}}$

However, ion-electron collisions may be waivered in comparison with ion-ion collisions. The two like-particle collision operators $C_{\mathrm{ee}}$ and $C_{\mathrm{ii}}$ may also be exchanged by operators similar to expression (8), videlicet

$$
\begin{aligned}
C_{j j}\left(f_{j}\right)= & v_{j j}(\kappa) \frac{q}{B} \frac{\partial}{\partial \mu} q \mu \frac{\partial}{\partial \mu} f_{j} \\
& +v_{j j}(\kappa) q F_{j} \frac{p_{j} m_{j}}{T_{j}}
\end{aligned}
$$

with

$$
p_{j}=\frac{\int \mathrm{d}^{3} v v_{j j}(\kappa) q f_{j}}{\frac{m_{j}}{T_{j}} \int \mathrm{d}^{3} v v_{j j}(\kappa) q^{2} f_{j}}
$$

An explanation of this form is that it may be taken from the Rosenbluth-McDonald-Judd form for distributions $f_{j}$ localized in velocity space, as in the current case. The term containing $\mathrm{p}$ certifies momentum conservation but may be waivered here, and for a localized distribution, its portion is small. This term is needed in the axial symmetric case where momentum conservation plays a significant role. Eventually, we need to define the collision frequencies as:

$v_{j k}(\kappa)=\frac{\sqrt{2} \pi n e^{4} \lambda}{m_{j}^{1 / 2} T_{j}^{3 / 2}} A_{j k}\left(x_{j}\right)$

where $x_{j}(\kappa)=m_{j k} / T_{j}$,

$A_{j k}\left(x_{j}\right)=\left(\eta_{k}+\eta_{k}^{\prime}-\frac{\eta_{k}}{2 x_{k}}\right) x_{j}^{-3 / 2}$

With

$\eta_{k}\left(x_{k}\right)=\frac{2}{\sqrt{\pi}} \int_{0}^{x_{k}} \mathrm{e}^{-t} t^{1 / 2} \mathrm{~d} t, \eta_{k}^{\prime}=\frac{d \eta}{d x_{k}}$

obviously in $A_{\mathrm{ei}}, x_{\mathrm{i}} \gg x_{\mathrm{e}}$, therefore

$A_{\mathrm{ei}}\left(x_{\mathrm{e}}\right)=x_{\mathrm{e}}^{-3 / 2}$

Coming back to the solution of kinetic equation (5), we need to consider the slow alteration in $\theta$ over a ripple period by using the field line equation $\theta=\theta_{0}+t \phi$, where $\theta_{0}$ is a reference angle for each field line. Then, by introducing the variable $\theta_{0}$ clearly into the basic kinetic equation, the first term can be expressed as:

$\sigma q \vec{n} \cdot \nabla f_{j}=\frac{\sigma q}{R} \frac{\partial f_{j}}{\partial \phi}$

and the second term is

$$
\begin{aligned}
\vec{v}_{\mathrm{d}} \cdot \nabla F_{j}= & -\frac{B_{0}}{\omega_{c_{j}}}\left(\frac{q^{2}+\mu B}{B}\right) \frac{1}{R} \\
& \times \frac{\partial F_{j}}{\partial r} \sin \left(\theta_{0}+t \phi\right)+O\left(\varepsilon^{2}\right)
\end{aligned}
$$

We look for a solution for those particles trapped in a magnetic field ripple, and for these, we expend Eq. (5) in $v_{\text {eff }} / \omega_{b}$. The term $\sigma q / R \partial f_{j} / \partial \phi$ could be expunged by operator $\sum_{\sigma} \int_{\phi_{1}}^{\phi_{2}} \frac{\mathrm{d} \phi}{q}$ where $q\left(\phi_{1}\right)=q\left(\phi_{2}\right)$. Unless, we make the ansatz

$f=\frac{\omega_{b}}{v_{\mathrm{eff}}} i^{(-1)}+f^{(0)}+\frac{\nu_{\mathrm{eff}}}{\omega_{b}} f^{(1)}+\cdots$

We get an inconsistence since

$\sum_{\sigma} \int_{\phi_{1}}^{\phi_{2}} \frac{\mathrm{d} \phi}{q} \vec{v}_{d_{j}} \cdot \nabla F_{j} \neq 0$ 
with the expansion (15) we see in the lowest order

$\frac{\partial f_{j}^{(-1)}}{\partial \phi}=0$

And in the first order, after applying the annihilator, one gets the following equation for $f_{j}^{(-1)}$ :

$$
\begin{gathered}
-\frac{\partial F_{j}}{\partial r} \frac{B_{0}}{\omega_{c o_{j}}^{R}} \int_{\phi_{1}}^{\phi_{2}} \frac{\mathrm{d} \phi\left(q^{2}+\mu B\right)}{q} \sin \left(\theta_{0}+t \phi\right) \\
\quad=\sum_{\kappa} v_{j \kappa}(\kappa) \frac{\partial}{\partial \mu} \frac{\mu}{B} J \frac{\partial f_{j}^{(-1)}}{\partial \mu}
\end{gathered}
$$

where

$$
\begin{aligned}
J & \equiv \int_{\phi_{1}}^{\phi_{2}} q \mathrm{~d} \phi \\
& =\int_{\phi_{1}}^{\phi_{2}} \sqrt{2\left[\kappa-\mu B\left(1-\delta \cos N \phi-\varepsilon \cos \left(\theta_{0}+t \phi\right)\right)\right]} \mathrm{d} \phi
\end{aligned}
$$

On the left-hand side of Eq. (17), considering $X=N \phi$ as the variable of integration with a range of less than $2 \pi$, we will have

$\sin \left(\theta_{0}+t \phi\right)=\sin \left(\theta_{0}+\frac{t}{N} X\right) \sim \sin \theta_{0}+O\left(\frac{1}{N}\right)$

And since $\frac{\partial(\mu J)}{\partial \mu}=-\int_{\phi_{1}}^{\phi_{2}} \frac{\mathrm{d} \phi}{q}\left(\mu B-q^{2}\right)$.

And $\frac{q^{2}}{\mu B} \sim O(\delta)$.

Equation (17) is as follows:

$$
\begin{aligned}
& \frac{\partial F_{j}}{\partial r} \frac{1}{\omega_{c o_{j}}^{R}} \sin \theta_{0} \frac{\partial}{\partial \mu} \mu J \\
& \quad=\frac{\partial}{\partial \mu} \frac{\mu}{B_{0}} J \frac{\partial f_{j}^{(-1)}}{\partial \mu}\left(\sum_{\kappa} v_{j \kappa}(\kappa)\right) O\left(\delta, \frac{1}{N}\right)
\end{aligned}
$$

The suitable solution of this equation in the range of $\frac{\kappa}{B_{\max }}<\mu<\frac{\kappa}{B_{\min }}$ is

$f_{j}^{(-1)}=\frac{\partial F_{j}}{\partial r} \frac{m_{j}}{e_{j}} \frac{1}{R} \sin \theta_{0}\left(\mu-\frac{\kappa}{B_{\max }}\right) \frac{1}{\sum_{\kappa} v_{j \kappa}(\kappa)}$

where $B_{\max }$ is given by [10]

$$
\begin{aligned}
\frac{B_{\max }}{B_{0}}= & 1-\varepsilon \cos \theta_{0}+\delta\left\{\sqrt{1-\alpha^{2} \sin ^{2} \theta_{0}}\right. \\
& \left.-\alpha\left|\sin \theta_{0}\right|\left[\pi-\sin ^{-1}\left(\alpha\left|\sin \theta_{0}\right|\right)\right]\right\}
\end{aligned}
$$

\section{Diffusion and heat transport}

The particle diffusion flux is defined as

$\Gamma_{j}=\int_{0}^{2 \pi} \frac{\mathrm{d} \theta}{2 \pi} \int_{0}^{2 \pi} \frac{\mathrm{d} \phi}{2 \pi} \sum_{\sigma} \int_{0}^{\infty} 2 \pi \mathrm{d} \kappa \int_{\kappa / B \max }^{\kappa / B} \frac{B d \mu}{q} f_{j}^{(-1)} \nu \mathrm{d} r_{j}$,

and the ion heat flux is defined as

$Q_{i}=\int_{0}^{2 \pi} \frac{\mathrm{d} \theta}{2 \pi} \int_{0}^{2 \pi} \frac{\mathrm{d} \phi}{2 \pi} \sum_{\sigma} \int_{0}^{\infty} 2 \pi \mathrm{d} \kappa \int_{\kappa / B \max }^{\kappa / B} \frac{B d \mu}{q} f_{i}^{(-1)} m_{i} \kappa \nu \mathrm{d} r_{i}$

where $\mathbf{v} d r_{j}=-\frac{\kappa}{\omega_{c o_{j}} R} \sin \theta_{0}$ and

$F_{j}=\left(\frac{m_{j}}{2 \pi T_{j}(r)}\right)^{3 / 2} n_{j}(r) \exp \left(\frac{m_{j} \kappa}{T_{j}(r)}\right), x_{j}=m_{j k} / T_{j}, \kappa=v^{2} / 2$

And

$f_{j}^{(-1)}=\frac{\partial F_{j}}{\partial r} \frac{m_{j}}{e_{j}} \frac{1}{R} \sin \theta_{0}\left(\mu-\frac{\kappa}{B_{\max }}\right) \frac{1}{\sum v_{j k}(\kappa)}$

$\Gamma_{j}=-\frac{A}{(2 \pi)^{3 / 2}}\left(\frac{T_{j}}{e B R}\right)^{2} \int_{0}^{\infty} \frac{\mathrm{d} x_{j} x_{j}^{5 / 2} e^{-x_{j}}}{\sum_{k} v_{j k}\left(x_{j}\right)}$

$$
\left[\frac{n_{j}^{\prime}}{n_{j}}+\frac{e_{j} \Phi^{\prime}}{T_{j}}-\left(\frac{3}{2}-x_{j}\right) \frac{T_{j}^{\prime}}{T_{j}}\right]
$$

And

$Q_{i}=-\frac{A}{(2 \pi)^{3 / 2}} T_{i}\left(\frac{T_{i}}{e B R}\right)^{2} \int_{0}^{\infty} \frac{\mathrm{d} x_{i} x_{i}^{7 / 2} e^{-x_{i}}}{\sum_{k} v_{i k}\left(x_{i}\right)}\left[\frac{n_{i}^{\prime}}{n_{i}}+\frac{e_{i} \Phi^{\prime}}{T_{i}}-\left(\frac{3}{2}-x_{i}\right) \frac{T_{i}^{\prime}}{T_{i}}\right]$

where $A=-\frac{2 \sqrt{2} N}{3 \pi} \int_{0}^{2 \pi} \mathrm{d} \theta_{0} \sin ^{2} \theta_{0} \int_{a}^{b} \mathrm{~d} \phi\left(1-\frac{B}{B \max }\right)^{3 / 2}, \Phi^{\prime}=$ $-\frac{T_{i}}{e}\left[\frac{n^{\prime}}{n}+3.37 \frac{T_{i}^{\prime}}{T_{i}}\right][10,12]$

Basically, collisional transport in tokamak depends on toroidal effects. In toroidal geometry, the particle and heat flux are greater than their values in the cylinder. Regardless of the magnetic field ripple effect in neoclassical transport, we can write the following equation for particle and energy flux:

$\Gamma_{p}=-D \frac{\partial n}{\partial r}+v n, \quad q_{E}=-n \chi \frac{\partial T}{\partial r}+v n T$

[13].

By using Eq. (2) in Sect. 2, for diffusion coefficient $D$, when $v>\delta \omega_{E}$ and $\omega_{E}=E_{r} / r B$, the radial excursion of a 
ripple banana is limited by collisions and Eq. (2) is still true. When $\omega_{E}(\varepsilon T / e)^{4}<\delta \omega_{E}<\nu$, the ripple diffusion is

$D_{R} \sim \varepsilon^{2}\left(\frac{\nu}{r}\right)^{1 / 2}\left(\frac{T}{e E_{r}}\right)^{3 / 2}\left(\frac{T}{e B}\right)^{1 / 2}$

The most complete expressions for the ambipolar diffusion and heat transport by assumption $\alpha<<1$ can be written as follows:

$$
\begin{aligned}
\Gamma_{a}= & -\frac{4.1}{v_{e i}} \delta^{3 / 2}\left(\frac{T_{e}}{e B R}\right)^{2} \\
& \times\left[\left(1+\frac{T_{i}}{T_{e}}\right) \frac{d n}{d r}+\frac{7}{2} \frac{n}{T_{e}} \frac{\mathrm{d}}{\mathrm{d} r}\left(T_{e}+T_{i}\right)\right] \\
Q_{i}= & -\frac{40.6}{v_{i i}} \delta^{3 / 2}\left(\frac{T_{i}}{e B R}\right)^{2} n \frac{d T_{i}}{d r_{e}}
\end{aligned}
$$

and

$v_{j i}=\frac{4}{3} \frac{\sqrt{2 \pi}}{m_{j}^{1 / 2} T_{j}^{3 / 2}} \ln \Lambda$

where $\Gamma_{a}$ is mean particle flux per unit area of either species across a magnetic surface and $Q_{i}$ is the mean ion heat flux or thermal diffusivity.

The simple form for radial profiles of tokamaks can be considered as follows: $n(r)=n_{0}\left(1-x^{2}\right), T_{i}=T_{i 0}\left(1-x^{2}\right)$, $\delta(r)=\delta_{a} x^{2}, x=r / a[1]$.

The ratio of particle and heat transport for the ripple contribution can be written as follow s: $\quad \chi_{i R} / D_{R} \sim 2\left(m_{i} / m_{e}\right)^{1 / 2}\left(T_{i} / T_{e}\right)^{7 / 2}\left(1+T_{i} / T_{e}\right)^{-1}$ while for toroidal contributions can be written as: $\chi_{i b} / D_{b} \sim 0.5\left(m_{i} / m_{e}\right)^{1 / 2}\left(T_{e} / T_{i}\right)^{7 / 2}\left(1+T_{i} / T_{e}\right)^{-1}$. In the condition of present tokamaks where $T_{i} / T_{e} \sim 0.5$, the $\chi_{i R}$ is relatively less important than that of in the condition which is $T_{i}=T_{e}$.

This difference in the results is due to the difference between values of $T_{i} / T_{e}$ [14].

\section{Experimental setup and results}

IR-T1 is an air-core transformer-type tokamak with a circular cross section, low beta and large aspect ratio, which has two stainless steels grounded fully, poloidal limiters and ohmic heating discharge system. The average pressure before discharge is in the range of $2.5-2.9 \times 10^{-5}$ Torr. It contains magnetic, electric, rake Mach probes and Mirnov coils for plasma diagnostics. Also it contains toroidal coils, ohmic coils and central solenoid and vertical coils.
Table 1 Some of IR-T1 tokamak characteristics

\begin{tabular}{ll}
\hline Parameters & Value \\
\hline Major radius $(r)$ & $0.45 \mathrm{~m}$ \\
Minor radius $(a)$ & $0.125 \mathrm{~m}$ \\
Material of first wall & Stainless steel \\
Limiter type and diameter $(m)$ & Ring limiter and 0.250 \\
Ripple at plasma edge and number of coils & 16 \\
Toroidal magnetic field $\left(B_{\mathrm{t}}\right)$ & $0.6-0.9 \mathrm{~T}$ \\
Plasma current $\left(I_{\mathrm{p}}\right)$ & $\mathrm{Up} \mathrm{to} 40 \mathrm{kA}$ \\
Discharge duration & $<35 \mathrm{~ms}$ \\
Energy confinement time & $1-3 \mathrm{~ms}$ \\
Electron density & $0.7-1.5 \times 10^{13} \mathrm{~cm}^{-3}$ \\
$V_{\text {Loop }}$ & $2.6-8 \mathrm{~V}$ \\
\hline
\end{tabular}

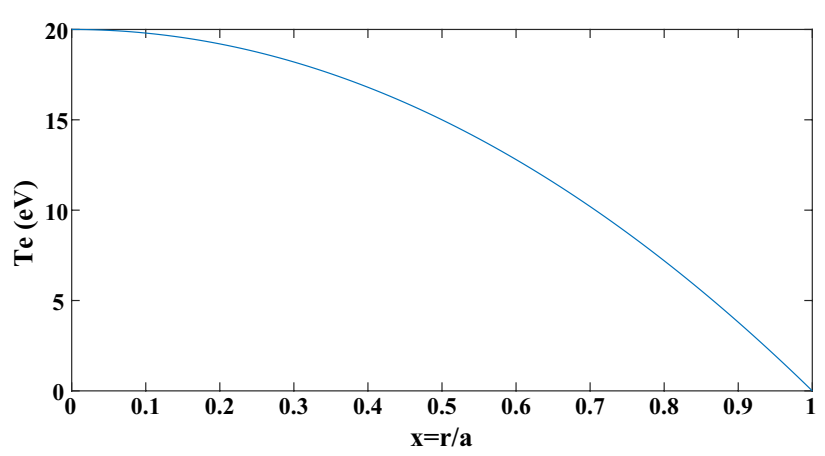

Fig. 1 Radial profiles of electron temperature versus $x=r / a$ for IR-T1 tokamak parameters

In IR-T1, 16 toroidal field coils produce a magnetic field around the torus to confine the plasma.

The experiments were performed in the hydrogen [15]. Some of the main characteristics of IR-T1 are shown in Table 1 [16].

The simple shape of the radial profiles of temperature and density is plotted according to Sect. 4, formulas. We observed the reduction as we move away from the center and move toward the plasma edge (Figs. 1, 2, 3, 4).

The ripple ratio $\delta_{B}$ (magnitude of the magnetic field ripple) $\delta_{B}=\left(B_{\max }-B_{\min }\right) /\left(B_{\max }+B_{\min }\right)$ for IR-T1 with 16 coils is calculated as $\delta_{B} \simeq 0.6$. For TorSopra, $\delta_{B}=0.5$, for JET, $\delta_{B}=0.08$, for ASDEX, $\delta_{B}=0.6$ and for JT060u $\delta_{B}=1[17]$.

Losses due to particle and energy transport can create many limitations in the design of the reactor magnet. Of course the number of toroidal coils can affect the dissipation of energy. The amplitude of the ripples is clearly lower when the number of toroidal coils is higher. For example, in JET tokamak, by reducing the number of coils from 32 to 16 , the ripples level increased from 1 to $10 \%$ [18-24]. 


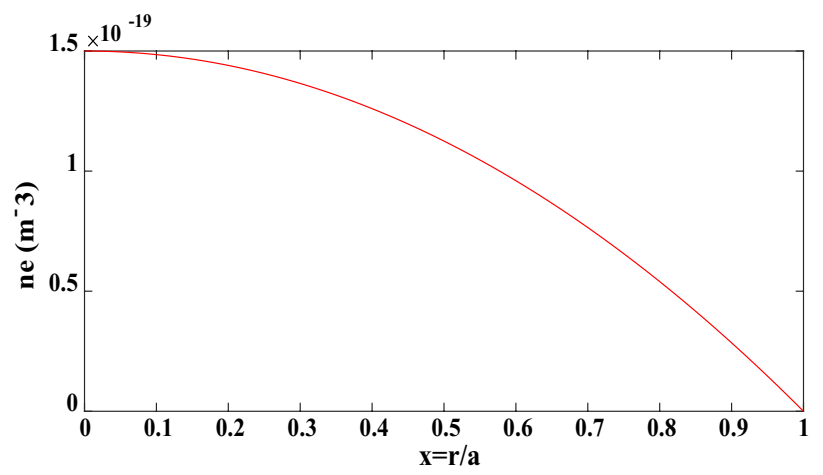

Fig. 2 Radial profiles of density versus $x=r / a$ for IR-T1 tokamak parameters

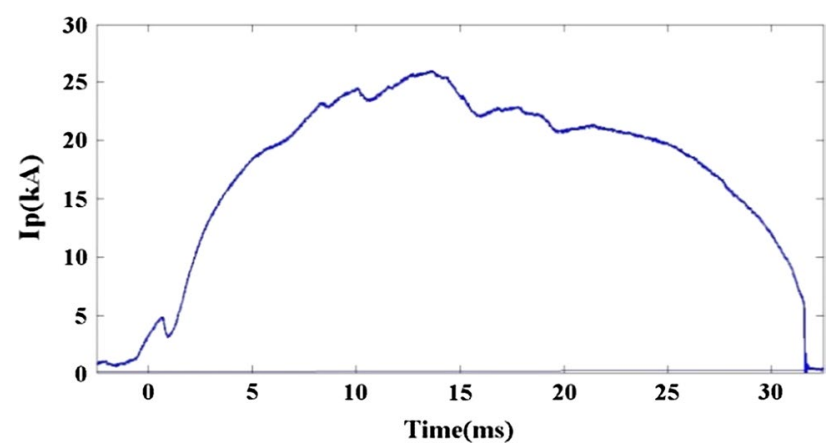

Fig. 3 Plasma current of IR-T1 tokamak

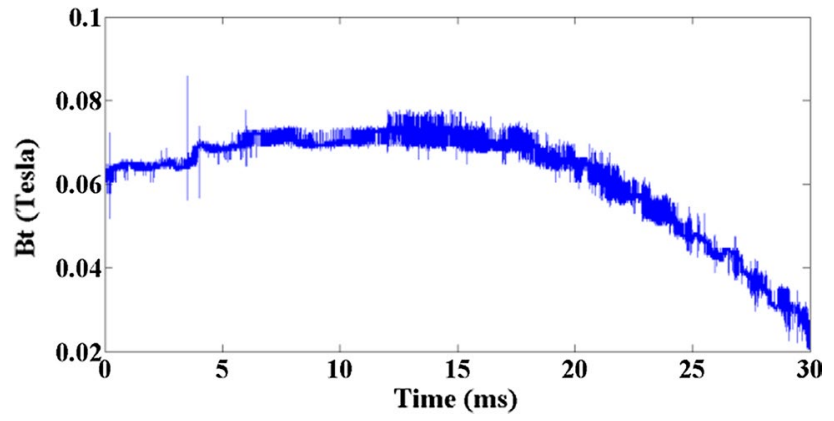

Fig. 4 Toroidal magnetic field $\left(B_{\mathrm{t}}\right)$ of IR-T1 tokamak

\section{The variation in transport coefficients on IR-T1 tokamak}

By using Eqs. (28) and (24), (30) in Sect. 4, for ripple and neoclassical transport, the coefficients have been evaluated numerically for IR-T1 tokamak parameters. The variations in the diffusion coefficient and ion heat conductivity with radius for IR-T1 parameters are plotted in Figs. 5 and 6, respectively.

As we see in Figs. 5 and 6 in the IR-T1 tokamak conditions, particle diffusion coefficient and heat conductivity do not differ

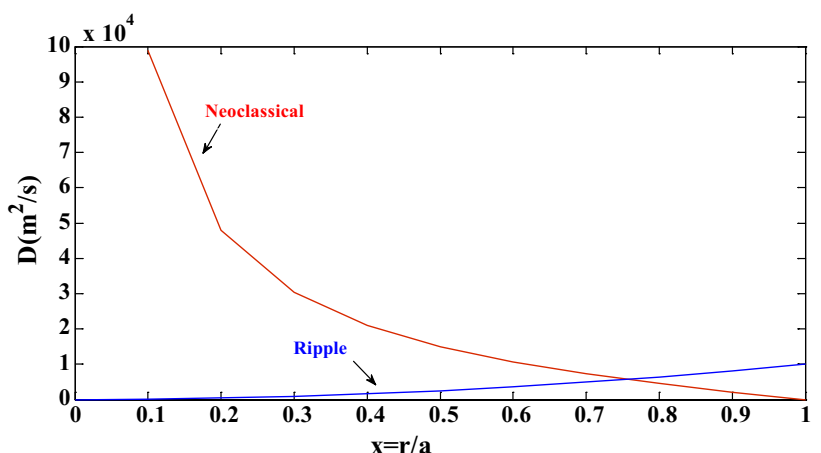

Fig. 5 Comparison of diffusion coefficient due to neoclassical and ripple transport versus radius, $x=r / a$ on IR-T1 tokamak

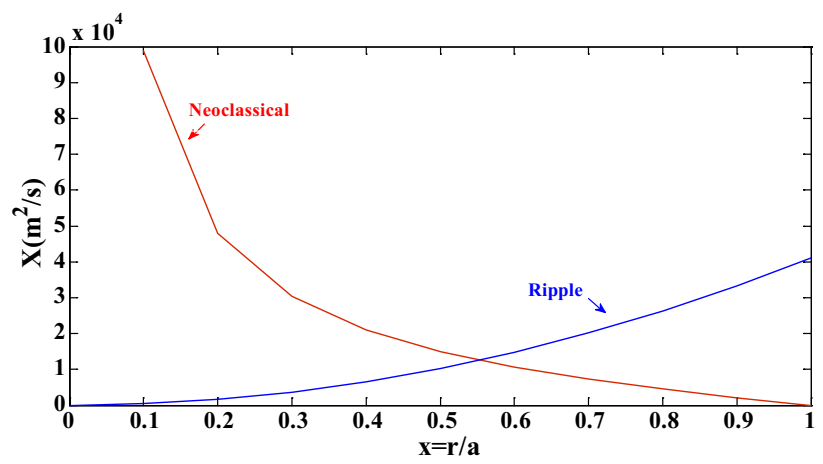

Fig. 6 Comparison of ion heat conductivity due to neoclassical and ripple transport versus radius, $x=r / a$ on IR-T1 tokamak

greatly. On both graphs, the neoclassical coefficient is decreasing by radius but the ripple distribution is increasing.

Also comparison of the plots shows that the thermal conductivity coefficient increases by increasing the radius, but this increase is faster than the diffusion coefficient with a factor about 4.1.

\section{Conclusion}

In this work, we investigated transport coefficients (diffusivity and ion heat conductivity) considering the effects of ripples in the magnetic field. In addition to the neoclassical diffusion, we observed the ripple diffusion and compared the transport coefficients for these two modes. The ripple ratio is also calculated numerically, $\delta_{B} \simeq 0.6$, for IR-T1 tokamak 
magnetic field. The observations indicate that diffusivity and ion heat conductivity decrease, as we move away from the plasma center in neoclassical mode. But in ripple mode, transport coefficients increase with the radius, and the rate of growth of the conductivity coefficient is greater than the diffusion coefficient.

Open Access This article is distributed under the terms of the Creative Commons Attribution 4.0 International License (http://creativeco mmons.org/licenses/by/4.0/), which permits unrestricted use, distribution, and reproduction in any medium, provided you give appropriate credit to the original author(s) and the source, provide a link to the Creative Commons license, and indicate if changes were made.

\section{References}

1. Stringer, T.E.: Effect of the magnetic field ripple on diffusion in Tokamaks. Nucl. Fusion 12, 694-698 (1973)

2. Khan, M., Schoepf, K., Goloborod'Ko, V.: Symplectic simulations of radial diffusion of in Tokamaks in the presence of TF ripples and a Neoclassical tearing mode. J. Fusion Energy 31(6), 547-561 (2012). https://doi.org/10.1007/s10894-011-9503-3

3. Wesson, J.: Tokamaks, pp. 374-389. Oxford press, Oxford (1997)

4. Khan, M., Schoepf, K., Goloborod'Ko, V.: Sympletic simulations of radial diffusion of fast Alpha particles in the presence of low-frequency modes in rippled Tokamaks. J. Fusion Energy 36, 40-47 (2017). https://doi.org/10.1007/s10894-016-0120-z

5. Khan, M., Zfar, A., Kamran, M.: Fast ion trajectory calculation using symplectic integration algorithm. J. Fusion Energy 34(2), 298-304 (2014). https://doi.org/10.1007/s10894-014-9801-7

6. Mimata, H., Tani, K., Tsutsui, H., et al.: Numerical study of the ripple resonance diffusion of Alpha particles in Tokamaks. Plasma Fusion Res. 4, 008 (2009)

7. Koskela, T., Akaslompolo, S.: Magnetic ripple and its implications in ITER design. In: Tekes Seminar Tampere. Aalto University School of Science and Technology, 2-3 June 2010

8. Gurevich, A.V., Dimant, Y.S.: Kinetic theory of the convective ripple transport in Tokamaks. Nucl. Fusion 21, 159-173 (1981)

9. Paul, E.J., Landreman, M., Poli, F.M., et al.: Rotation and neoclassical ripple transport in ITER. Nucl. Fusion 57, 116044 (2017)

10. Connor, J.W., Hastie, R.J.: Neoclassical diffusion arising from magnetic-field ripples in Tokamaks. Nucl. Fusion 13, 221-225 (1973)

11. Stringer, T.E.: Effect of the magnetic field ripple on diffusion in tokamaks. Nucl. Fusion 12, 689 (1972)
12. Dervlak, M., Heyn, M.F., Kalyuzhnyj, V. N., et al.: Effective ripple for the W7-X magnetic field calculated by the PIES code. In: 30th EPS Conference on Controlled Fusion and Plasma Physics, St. Petersburg, 7-11 July 2003, ECA, vol 27A, p. 1.12 (2003)

13. Baker, D.R., Wade, M.R., Petty, C.C., et al.: Particle transport phenomena in the DIII-D tokamak. Nucl. Fusion 40(5), 1003-1011 (2000)

14. Kadomtsev, B.B., Pogutse, O.P.: Trapped particles in toroidal magnetic systems. Nucl. Fusion 11, 67 (1971)

15. Ghanbari, M., Ghoranneviss, M., Mohammadi, M., et al.: Runaway electron generation decrease during a major disruption by limiter biasing in tokamaks. Radiat. Effects Defects Solids 168, 664-674 (2013)

16. Hajakbari, F., Hojabri, A., Ghoranneviss, M.: Study of runaway electrons in the Iran Tokamak 1(IR-T1). In: 33rd EPS Conference on Plasma Physics, Rome, 19-23 June 2006 ECA, vol. 30I, D-5.020 (2006)

17. Bunno, M., Nakamura, Y., Suzuki, Y., et al.: The finite beta effects on the toroidal field ripple in a tokamak plasma. Plasma Sci. Technol. 15(2), 115-118 (2013)

18. Garbet, X., Mantica, P., Angioni, C., et al.: Physics of transport in tokamaks. Plasma Phys. Control. Fusion 46(12B), B557 (2004)

19. Davidson, J.N.: Effect of toroidal field ripple on particle and energy transport in tokamak. Nucl. Fusion 16(5), 731 (1976)

20. Belyaev, N.R., Girka, I.O., Gritsyna, V.T.: Effect of periodic ripple in an axial confining magnetic field on the Alfven heating of a cylindrical plasma. Plasma Phys. Rep. 29(5), 432-439 (2003)

21. Chen, F.F.: Introduction to Plasma Physics and Controlled Fusion, vol 1, Plasma Physics, pp 186-194 (1974)

22. Paul, E.J., Landerman, M., Poli, F.M.: Rotation and neoclassical ripple transport in ITER. Nucl. Fusion 57, 116044 (2017)

23. Nave, M.F.F., Johnson, T., Eriksson, L.-G.: The influence of magnetic field ripple on JET Intrinsic Rotation. In: 36th EPS Conference on Plasma Phys. Sofia, June 29-July 3, 2009 ECA, vol. 33E, p. 2.168 (2009)

24. Mahdavipour, B., Salar Elahi, A., Ghoranneviss, M.: Effects of resonant helical field on toroidal field ripple in IR-T1 Tokamak. In: IOP Conference Series: Journal of Physics, Conference Series, vol. 982, p. 012004 (2018). https://doi.org/10.1088/17426596/982/1/012004

Publisher's Note Springer Nature remains neutral with regard to jurisdictional claims in published maps and institutional affiliations. 